\section{The hospice: a place to die or just passing on?}

\author{
Bruce A. Foggo
}

\section{St Joseph's Mercy Hospice, Auckland, New Zealand}

A consequence of increasing medical specialisation is that some patients with life-threatening illness, but not imminently dying, may require transfer to another institution for continuing care. A qualitative reflective study was carried out over 9 months to observe the process that resulted in a decision to transfer a hospice patient for care elsewhere. Observations about the course of patients were recorded in a journal and as separate crossreferenced entries for individual patients. The journal entries were reviewed to determine whether a consistent decision process could be identified and for emerging themes that reflected the decision process. This paper reviews the observed decision process and explores the tensions that became apparent between the traditional 'care of the dying' role and a perceived 'medicalisation' of care at St Joseph's Mercy Hospice, Auckland, New Zealand.

Keywords: Hospice, medicalisation, care of the dying

The modern hospice has it is origin in the traditions of the medieval refuge providing shelter for travellers. This role extended to care of the dying and in its modern counterpart to care of the dying with appropriate medical support. The advent of the modern hospice has seen a move towards increasing medical specialisation and concern that the hos-

\section{Address for correspondence: \\ Dr Bruce A. Foggo MB ChB FAChPM, Palliative Medicine Specialist, St Joseph's Mercy Hospice, PO Box 9911, Newmarket, Auckland 1031, New Zealand Tel: +64 9 6239761; Fax: +64 96239751 E-mail: brucef@mercy.co.nz}

pice will become but one more specialist medical facility and lose its role as a provider of holistic care for dying patients. The tensions between these roles are apparent locally and are being challenged in the international palliative care literature (1-3).

St Joseph's Mercy Hospice provides community and in-patient palliative care services for the central Auckland area. The patient catchment is approximately 500,000. The in-patient unit has 15 beds. Community services are provided by a nursing team based at the hospice. At any time, there are about 150-200 patients receiving care from the community nursing team.

With demands on beds, the hospice is not able to provide long-term care and is frequently faced with the prospect of transferring patients to alternative care in a nursing home or aged-care facility.

This study reflects on the process and outcome of decisions made within the hospice that led to a patient remaining in the hospice or transferring to alternative care. The aim was to review our practice critically and determine whether a consistent and legitimate decision process could be identified.

\section{PATIENTS AND METHODS}

A journal was kept over the period 20 March to 30 August 2000 , which recorded observations about process and decision-making for the care of patients in the hospice who were not imminently dying.

Observations about the course of individual patients were recorded in the journal proper and as separate crossreferenced entries for individual patients. Patients included were those for whom there was any suggestion that continuing care in the hospice or alternative care may be required. A patient became 'eligible' for inclusion in the study at the time that this became more than a 'suggestion'. Both informal and formal process was noted. Entries were made daily, where possible, and at least several entries were made each week.

The methodological approach to the analysis of the journal entries was critical reflection and thematic analysis (4). Key themes which emerged that related to the decision process across several entries were identified and clustered together.

\section{Decision process}

An attempt was made to identify the process that led to a decision being made regarding future care for that patient. 
The decision outcomes were: (i) continued care as an inpatient at the hospice; (ii) transfer to a nursing home or aged-care facility; and (iii) discharge for care at home.

An attempt was made to classify the process into one of four categories:

1. Consensus decision - general agreement either formal or informal at a team andlor family meeting that a decision or plan was appropriate.

2. Medical decision - a decision or initiative made by the medical staff without wider consultation.

3. Family request - a direct request from family.

4. Informal decision - no identifiable decision process.

\section{RESULTS}

A summary of the results obtained is given in Table 1.

\section{DISCUSSION}

While there is no one clear process for determining who gets continuing care in the hospice, it is apparent from the survival time figures for the three outcome groups (hospice/nursing home or aged-care facility/home) that appropriate decisions were being arrived at based on survival times alone. Apart from a shorter life expectancy for hospice patients, perhaps indicating that this group represented a 'sicker' group, there were no major differences between groups that identified these patients as requiring continued hospice care and there was no quantitative assessment or measurement of need for continued hospice care.

The major underlying theme that emerged from the study was that of uncertainty. This included uncertainty about the role of the hospice and uncertainty about the decision processes within the hospice. This resulted in lack of clarity of expectation for patients, families and staff. Underlying this was a lack of clarity or description of the decision process for patients requiring continuing care in the hospice or elsewhere. This is reflected in the four decision process categories and particularly that in 8 of 30 patients no actual decision process could be identified.

This uncertainty led to a further theme of tension which was apparent at a number of levels. Tension between the traditional 'care of the dying' role and perceived 'medicalisation' of care was apparent in discussions about individual patients and in wider discussions at ward meetings and was an underlying theme of informal 'talk' on the ward. While there has not been an overt change in the hospice role, there was an apparent impression that priority was increasingly being given to acute medical admissions over patients requiring admission for psychosocial support or nursing care. What has become
Table 1. Summary of results

\begin{tabular}{lcc}
\hline PATIENT PROFILE & & \\
Number of patients reviewed & 30 & \\
Male & 9 & \\
Female & 21 & \\
Average age (years) & 65.4 & \\
& & Average age \\
(years) \\
OUTCOMES & & 65 \\
$\quad$ Remained in hospice & 16 & 66 \\
Transferred & 7 & 67 \\
Discharged home & 7 & \\
AVERAGE LENGTH OF TIME FROM & & \\
DECISION RE CARE TO DEATH & & \\
Hospice (days) & 21 & \\
Private hospital or home care (days) & 54 & \\
DECISION PROCESS & & \\
Consensus & 10 & \\
Medical initiative & 7 & \\
Family request & 5 & \\
Informal & 8 &
\end{tabular}

apparent is that peoples' concept of hospice care and attitudes are different and perhaps becoming more entrenched as they see the nature of care changing. There are some staff who recall the 'old' hospice (prior to it being known as a hospice) as a long-term care facility for the aged-infirm and dying and are less comfortable with their current role. These tensions extended not only to decisions about care but to who makes the decision about future care and the perceived dominance of medical priorities. This is reflected in the number of decisions which were made initially without wider consultation.

Concerns about equity and fairness were further themes that were apparent at team meetings and discussions about future care of patients - the fairness of looking after one patient long-term in the hospice against another who may be transferred and the equitable use of resources when there is a high demand from the community for admission to in-patient beds. While an attempt was made to invoke these concepts, there was no formal process to account for this.

Equity and fairness had overlap with a further theme of attachment. There was no doubt that some patients appeared more 'attractive' than others to nurse long-term with the risk that staff became unwitting advocates for these patients to remain in hospice care. And the longer nursed, the closer the attachment between staff, patient and families. The theme of attachment and its impact on professional carers is recognised in other health care settings (5) and, while often not openly acknowledged, it is inevitable that these issues will affect the decision process about future care for these patients.

Underlying much of the above was a further theme concerning rules - primarily, the lack of stated rules or criteria 
to guide decision making. This was apparent in many of the discussions about patient care when informal or historical rules were invoked to sway a decision one way or other. The lack of clarity about decision process and lack of rules underpinned the other themes of uncertainty and tension and the concerns about equity and fairness.

\section{CONCLUSIONS}

The major underlying theme of the study is one of uncertainty of expectations. This led to different expectation at all levels within the hospice and is reflected in the four different categories of decision process when considering future care options for patients in the hospice who are not imminently dying. While the decision processes apparently identified a group of sicker patients who had a lesser life expectancy and remained in the hospice, there was no formal process in place that confirmed this was the case. This led to some tensions regarding application of assumed rules and concerns about equity and fairness.

In acknowledging the above, there is a risk that the hospice by virtue of its public positioning may over-sell itself and promote expectations of a service which may not always be met. Where this expectation results in dissatisfaction with a perceived commitment (care in the hospice for the duration of final illness) not being met, there is a real danger that the standing of the service will be compromised in the public eye. Similar concerns are raised by Fallon and Dunlop (6) in an editorial which questioned present directions in training for, and delivery of, palliative care services. The major recommendation that emerged from the study was the introduction of strategies across the hospice organisation to reduce uncertainty.
These strategies would include:

1. A clearer understanding of the role of the hospice.

2. An explicit acknowledgement that the hospice is not able to provide long-term care.

3. A tighter definition and awareness of staff roles, responsibilities and expectations at all levels.

4. A commitment to developing criteria for continued care or transfer and a transparent decision process relating to this.

5. A need to acknowledge the capability of care providers other than the hospice, and support for them in their role of providing care for terminally ill patients.

\section{REFERENCES}

1. Barbato M. Palliative care in the 21 st century - sink or swim. Aust NZ Soc Palliat Med Newsletter 1999;8:2-4.

2. Kearney M. Palliative medicine - just another specialty? Palliat Med 1992;6:9-46.

3. Praill D. Who are we here for? [Editorial]. Palliat Med 2000;14:91-2.

4. Ritchie J, Spencer LC. Qualitative data analysis for applied policy research. In: Bryman A, Burgess K. (eds) Analysing Qualitative Data. London:Routledge, 1994.

5. Main FT. The ailment. Br J Med Psychiatry 1957;30:129-45.

6. Fallon M, Dunlop R. Palliative care - or is it? [Editorial]. Palliat Med 2002;16:1-3. 